PROCEEDINGS OF THE

AMERICAN MATHEMATICAL SOCIETY

Volume 128, Number 2, Pages 439-444

S 0002-9939(99)05386-1

Article electronically published on September 24, 1999

\title{
A FATOU THEOREM FOR THE EQUATION $u_{t}=\Delta(u-1)_{+}$
}

\author{
MARIANNE K. KORTEN \\ (Communicated by Christopher D. Sogge) \\ Dedicated to the memory of Eugene Fabes
}

\begin{abstract}
In one space dimension and for a given function $u_{I}(x) \in C_{0}^{\infty}$ (say such that $u_{I}(x)>1$ in some interval), the equation $u_{t}=\Delta(u-1)_{+}$can be thought of as describing the energy per unit volume in a Stefan-type problem where the latent heat of the phase change is given by $1-u_{I}(x)$. Given a solution $0 \leq u \in L_{\text {loc }}^{1}\left(\mathbb{R}^{n} \times(0, T)\right)$ to this equation, we prove that for a.e. $x_{0} \in \mathbb{R}^{n}$, there exists $\lim _{(x, t) \in \Gamma_{\beta}^{k}\left(x_{0}\right),(x, t) \rightarrow x_{0}}(u(x, t)-1)_{+}=\left(f\left(x_{0}\right)-1\right)_{+}$, where $f=\partial \mu / \partial||$ is the Radon-Nikodym derivative of the initial trace $\mu$ with respect to Lebesgue measure and $\Gamma_{\beta}^{k}\left(x_{0}\right)=\left\{(x, t):\left|x-x_{0}\right|<\beta \sqrt{t}, 0<t<k\right\}$ are the parabolic "non-tangential" approach regions. Since only $(u-1)_{+}$is continuous, while $u$ is usually not, $\lim _{(x, t) \in \Gamma_{\beta}^{k}\left(x_{0}\right),(x, t) \rightarrow x_{0}} u(x, t)=f\left(x_{0}\right)$ does not hold in general.
\end{abstract}

\section{INTRODUCTION}

In this contribution we want to address the existence of pointwise limits of nonnegative solutions to the equation

$$
u_{t}=\Delta(u-1)_{+},
$$

where $(x, t) \in \mathbb{R}^{n} \times(0, T)$ when $(x, t)$ approaches the initial surface $\{t=0\}$. In one space dimension and for a given function $u_{I}(x) \in C_{0}^{\infty}$ (say such that $u_{I}(x)>1$ in some interval), equation (1.1) can be thought of as describing the energy per unit volume in a Stefan-type problem where the latent heat of the phase change is given by $1-u_{I}(x)$. Note that discontinuous solutions should be expected for (1.1) (see $[\mathrm{BKM}])$.

In previous papers $([\mathrm{AK}],[\mathrm{K}])$ a-priori regularity of non-negative solutions in the sense of distributions $u \in L_{\mathrm{loc}}^{1}\left(\mathbb{R}^{n} \times(0, T)\right)$ was found, mainly $u, \nabla_{x}(u-1)_{+}$and $\frac{\partial}{\partial t}(u-1)_{+} \in L_{\text {loc }}^{2}\left(\mathbb{R}^{n} \times(0, T)\right)$, and continuity of $(u-1)_{+}$in $\left(\mathbb{R}^{n} \times(0, T)\right)$. Also $(u-1)_{+}$is a (weak) subsolution to the heat equation. From the weak Harnack inequality

$$
\int_{\mathbb{R}^{n}} u(x, t) \exp \left(-c|x|^{2}\right) d x \leq M(u, n, T),
$$

Received by the editors February 28, 1998.

1991 Mathematics Subject Classification. Primary 35K65, 31A20.

This research was partially supported by PIDs 3668/92 and 3164/92-CONICET and EX 071UBA.

(C)1999 American Mathematical Society 
for some $c=c(T) \in \mathbb{R}_{+}$and $0<t<T / 2$ (see [AK]), existence of a unique initial trace follows, which is a Radon measure satisfying the growth condition at infinity

$$
\int_{\mathbb{R}^{n}} \exp \left(-c|x|^{2}\right) d \mu(x)<\infty,
$$

and is taken in the appropriate sense. Each such measure gives rise to a solution to $(1.1)$, which is unique (see $[\mathrm{K}]$ ).

Fatou theorems for solutions to degenerate parabolic equations were proven in [DFK] (for the porous medium equation $u_{t}=\Delta u^{m}, m>1$ ) and [H] (for generalized diffusions $u_{t}=\Delta \phi(u), \phi^{\prime}(u)>0$ for $u>0$ and growth conditions on $\phi$ near 0 and at infinity). In both situations the solutions $u$ are continuous (see [DFK], [DB]) and the result reads as follows:

Let $\Gamma_{\beta}^{k}\left(x_{0}\right)=\left\{(x, t):\left|x-x_{0}\right|<\beta \sqrt{t}, 0<t<k\right\}$ be the parabolic non-tangential approach "cones". For a.e. $x_{0} \in \mathbb{R}^{n}$, there exists $\lim _{(x, t) \in \Gamma_{\beta}^{k}\left(x_{0}\right),(x, t) \rightarrow x_{0}} u(x, t)=$ $f\left(x_{0}\right)$, where $f=\partial \mu / \partial||$ is the Radon-Nikodym derivative of the initial trace with respect to Lebesgue measure.

An analogous result holds for non-negative solutions of the heat equation; in this case the result follows from estimates on the heat kernel.

In this paper we prove the following

1.1. Theorem. Let $0 \leq u \in L_{\mathrm{loc}}^{1}\left(\mathbb{R}^{n} \times(0, T)\right)$ be a solution in the sense of distributions of (1.1). Let $\mu$ be its initial trace and $f=d \mu / d x$ the Radon-Nikodym derivative of $\mu$ with respect to the Lebesgue measure. Then for almost every $x_{0} \in \mathbb{R}^{n}$,

$$
\lim _{(x, t) \in \Gamma_{\beta}^{k}\left(x_{0}\right),(x, t) \rightarrow x_{0}}(u(x, t)-1)_{+}=\left(f\left(x_{0}\right)-1\right)_{+} .
$$

Theorem 1.1 is proven stepwise: In Section 2 we prove it for initial traces which are locally integrable functions satisfying (1.3). The proof uses a lemma which allows a reduction to the linear case due to Dahlberg, Fabes and Kenig (Lemma 2.2 from $[\mathrm{DFK}])$. Then we follow an argument of $[\mathrm{H}]$, getting the necessary equicontinuity of a scaled family of comparison functions from the results of DiBenedetto ([DB]) which applies to (1.1) due to a priori integrabilities of $u,(u-1)_{+}, \frac{\partial(u-1)_{+}}{\partial t}$ and $\nabla(u-1)_{+}$obtained in $[\mathrm{K}]$ and $[\mathrm{AK}]$. In Section 3 we prove the result if the initial trace is a measure satisfying (1.3). We regularize the singular part of the initial measure and use the convergence of the solutions thus obtained. Here the uniform integrability of suitable heat functions plays a strong role. Then we use the uniqueness result of $[\mathrm{K}]$ to complete the proof. In Section 4 we exhibit an example which shows that

$$
\lim _{(x, t) \in \Gamma_{\beta}^{k}\left(x_{0}\right),(x, t) \rightarrow x_{0}} u(x, t)=f\left(x_{0}\right)
$$

does not hold in general for solutions to (1.1).

I wish to thank Carlos Segovia for his encouragement and for many helpful discussions on the subject of this paper.

\section{Step 1: The initial trace $\mu$ IS A LOCAlly integrable function}

Let $0 \leq \mu=u_{I}(x) \in L_{\text {loc }}^{1}\left(\mathbb{R}^{n}\right)$, such that $u_{I}$ satisfies (1.3) and let $u(x, t)$ be the (unique) solution to (1.1) with initial trace $u_{I} ; u$ is defined in $\mathbb{R}^{n} \times(0, T)$ where $T=T\left(u_{I}\right)$, and $\left\|u(x, t)-u_{I}(x)\right\|_{L^{1}(K)} \rightarrow 0$ for any compact $K \subset \mathbb{R}^{n}$ (see $[\mathrm{AK}]$ ).

For $0<\alpha, h$ let $\Gamma_{\alpha}^{h}\left(x_{0}\right)=\left\{(x, t) \in \mathbb{R}^{n} \times(0, \infty):\left|x-x_{0}\right| \leq \alpha \sqrt{t}, 0<t<h\right\}$. 
2.1. Remark. Let $\alpha, h>0$. For a.e. $x_{0} \in \mathbb{R}^{n}$,

$$
0 \leq \liminf _{(x, t) \in \Gamma_{\alpha}^{h}\left(x_{0}\right),(x, t) \rightarrow x_{0}}(u(x, t)-1)_{+} \leq \lim _{t \rightarrow 0}(u(x, t)-1)_{+}=\left(u_{I}\left(x_{0}\right)-1\right)_{+} .
$$

This is a consequence of the convergence of $u(., t) \rightarrow u_{I}$ in $L_{\text {loc }}^{1}\left(\mathbb{R}^{n}\right)$, the Lipchitz continuity of $g(u):=(u-1)_{+}$, and the continuity of $(u-1)_{+}$in $\mathbb{R}^{n} \times(0, T)$. Therefore, for a.e. $x_{0} \in E_{0}:=\left\{x_{0} \in \mathbb{R}^{n}: u_{I}\left(x_{0}\right) \leq 1\right\}$,

$$
\lim _{(x, t) \in \Gamma_{\alpha}^{h}\left(x_{0}\right),(x, t) \rightarrow x_{0}}(u(x, t)-1)_{+}=0 .
$$

Here we write $u_{I}(x)$ for the precise representative of $u_{I}$; that is, we understand $u_{I}(x)=\lim _{r \rightarrow 0} \frac{1}{\left|B_{r}(x)\right|} \int_{B_{r}(x)} u_{I}(y) d y$.

2.2. Lemma. Let $E=\left\{x_{0} \in \mathbb{R}^{n}: u_{I}\left(x_{0}\right)>1\right\}$, and $0<\alpha<$ T. For a.e. $x_{0} \in E$,

$$
\lim _{(x, t) \in \Gamma_{1}^{\alpha}\left(x_{0}\right),(x, t) \rightarrow x_{0}} u(x, t)=u_{I}\left(x_{0}\right) .
$$

Proof. Given $\epsilon>0$, let $E_{\epsilon}=\left\{x_{0} \in \mathbb{R}^{n}:\left(u_{I}\left(x_{0}\right)-1\right)_{+}>\epsilon\right\}$. Then $E=\bigcup_{\epsilon>0} E_{\epsilon}$.

Let $E_{\epsilon}^{\prime}=\left\{\right.$ density points of $\left.E_{\epsilon}\right\}$. Let $x_{0} \in E_{\epsilon}^{\prime}$. Without loss of generality we may assume $x_{0}=0$ and $\alpha>1$.

Let $v_{I}=(1+\epsilon) \chi_{E_{\epsilon}}$, whence $v_{I} \leq u_{I}$ a.e. Let $v(x, t)$ be the solution of (1.1) evolving from the initial datum $v_{I}$. By the results in [B] and $[\mathrm{AK}] v(x, t) \leq u(x, t)$ for a.e. $(x, t) \in \mathbb{R}^{n} \times(0, T)$. For any $\lambda>0$, let $v_{\lambda}(x, t)=v\left(\lambda x, \lambda^{2} t\right), v_{\lambda}$ is a solution to (1.1). Let $d_{\lambda}$ be its initial trace. Following [H], Lemma 1.6, we find $d_{\mu_{\lambda}} \rightarrow(\epsilon+1)$ weakly. Consider the family $\left(v_{\lambda}(x, t)-1\right)_{+}=\left(v\left(\lambda x, \lambda^{2} t\right)-1\right)_{+}$. Since $\left\{v_{\lambda}\right\}$ is locally bounded in $\mathbb{R}^{n} \times(0, T)$ and for any compact set $K \subset \mathbb{R}^{n} \times(0, T)$ the norms $\left\|\left(v_{\lambda}-1\right)_{+}\right\|_{L^{2}(K)},\left\|\frac{\partial}{\partial t}\left(v_{\lambda}-1\right)_{+}\right\|_{L^{2}(K)}$, and $\left\|\frac{\partial}{\partial x_{i}}\left(v_{\lambda}-1\right)_{+}\right\|_{L^{2}(K)}$ can be estimated in terms of $\left\|\left(v_{\lambda}-1\right)_{+}\right\|_{L^{2}\left(K^{\prime}\right)}, K \subset K^{\prime} \subset \mathbb{R}^{n} \times(0, T), K^{\prime}$ compact (see $[\mathrm{AK}])$, from the results in $[\mathrm{DB}]$ the equicontinuity of $\left(v_{\lambda}-1\right)_{+}$on any compact subset of $\mathbb{R}^{n} \times(0, T)$ follows. Therefore there exists a subsequence (which we will still call $\left.\left(v_{\lambda}-1\right)_{+}\right)$converging uniformly on compact subsets of $\mathbb{R}^{n} \times(0, T)$. Now take $K=\bar{B}_{1}(0) \times\{1\}=\left\{(x, t) \in \mathbb{R}^{n} \times(0, T):|x| \leq 1, t=1\right\}$. We obtain

$$
v_{\lambda}\left(x^{\prime}, 1\right)>1+\frac{\epsilon}{2}, \quad \text { for }\left|x^{\prime}\right|<1
$$

if $\lambda<\lambda_{0}$, whence

$$
u(x, t) \geq v(x, t)>1+\frac{\epsilon}{2}, \quad|x|^{2}<t, t<\lambda_{0},
$$

and

$$
\liminf _{(x, t) \in \Gamma_{1}^{T}\left(x_{0}\right),(x, t) \rightarrow x_{0}}(u(x, t)-1)_{+}>0 \quad \text { for } \quad \text { a.e. } x_{0} \in E .
$$

In order to uniformize the height $h$ of the "non-tangential" approach parabolas, let us split $E_{\epsilon}$ into a countable family of sets $E_{\epsilon}^{k}$ as follows:

Given $x_{0} \in E_{\epsilon}$, there exists $h_{x_{0}}>0$ such that $u(x, t)>1+\frac{\epsilon}{2}$ in $\Gamma_{\alpha}^{h_{x_{0}}}\left(x_{0}\right)$. Now let $k=\min \left\{k: h_{x_{0}} \geq 2^{-k}\right\}$.

Let us recall that by a familiar point of density argument (see [C]), given $\alpha, \beta>$ $0, h>0, \epsilon>0$ and a closed set $F$, there exists a closed set $F_{0},\left|F \backslash F_{0}\right|<\epsilon$ and $k>0$ such that $\bigcup_{x_{0} \in F_{0}} \Gamma_{\beta}^{k}\left(x_{0}\right) \subset \bigcup_{x_{0} \in F} \Gamma_{\alpha}^{h}\left(x_{0}\right)$. 
Let $F \subset E$ be compact, and $R=\bigcup_{x_{0} \in F} \Gamma_{\alpha}^{h}$. Applying Lemma 2.2 from [DFK] in $R$ yields the existence and finiteness of the limit

$$
\lim _{(x, t) \in \Gamma_{\alpha}^{h}\left(x_{0}\right),(x, t) \rightarrow x_{0}} u(x, t) .
$$

Then, the convergence $\left\|u(., t)-u_{I}\right\|_{L^{1}} \rightarrow 0$ yields its equality to $u_{I}\left(x_{0}\right)$. (Alternatively, $u(x, t)$ solves the heat equation in $R$, whence its "non-tangential" limit equals $u_{I}\left(x_{0}\right)$, for a.e. point $x_{0} \in F$. )

\section{Step 2: The initial trace $\mu$ IS A Measure}

3.1. Lemma. Let $\mu=f+\mu_{s}$ be the initial trace of $u$, where $f$ is absolutely continuous and $\mu_{s}$ is singular with respect to Lebesgue measure.

For a.e. $x_{0} \in \mathbb{R}^{n}$,

$$
\lim _{(x, t) \in \Gamma_{\alpha}^{h}\left(x_{0}\right),(x, t) \rightarrow x_{0}}(u(x, t)-1)_{+}=\left(f\left(x_{0}\right)-1\right)_{+} .
$$

Proof. Take $\mu_{s}^{\epsilon}=\mu_{s} * \rho_{\epsilon}$, where $\rho_{\epsilon}$ are the usual compactly supported mollifiers. Writing $u_{g}$ for the solution of (1.1) with initial datum $g$, by comparison (see [B], $[\mathrm{AK}])$ we have

$$
u_{f} \leq u_{f+\mu^{\epsilon}}+\leq w_{\epsilon} \quad \text { a.e. in } \mathbb{R}^{n} \times(0, T),
$$

where $w_{\epsilon}$ is the solution of the heat equation with initial datum $f+\mu_{s}^{\epsilon}+1$. From the results in Section 2 we have

$$
\lim _{(x, t) \in \Gamma_{\alpha}^{h}\left(x_{0}\right),(x, t) \rightarrow x_{0}}\left(u_{f}(x, t)-1\right)_{+}=\left(f\left(x_{0}\right)-1\right)_{+}, \quad \text { a.e. in } \mathbb{R}^{n} .
$$

To show that $u_{f+\mu_{s}^{\epsilon}} \rightarrow u_{f+\mu}$ a.e. in $\mathbb{R}^{n} \times(0, T)$, let us recall that $\left\{u_{\epsilon}\right\}$ is bounded in $L^{2}(K)$, for any compact subset $K$ of $\mathbb{R}^{n} \times(0, T)$ ), whence a subsequence of $\left\{u_{\epsilon}\right\}$, which we call again $\left\{u_{\epsilon}\right\}$, can be chosen such that $\lim _{\epsilon \rightarrow 0} u_{\epsilon}=u$, and $\lim _{\epsilon \rightarrow 0}\left(u_{\epsilon}-1\right)_{+}=h(x, t)$, a.e. in $\mathbb{R}^{n} \times(0, T)$. To show that $h(x, t)=(u-1)_{+}$, take arbitrary $0<t_{1}<t_{2}<T, 0<R \in \mathbb{R}, B=B_{R}(0)$ and let $\epsilon \rightarrow 0$ in

$$
\begin{gathered}
\int_{t_{1}}^{t_{2}} \int_{B} u_{\epsilon} \chi_{\left\{u_{\epsilon}>1\right\}} \chi_{\{h>0\}}=\int_{t_{1}}^{t_{2}} \int_{B}\left(u_{\epsilon}-1\right) \chi_{\left\{u_{\epsilon}>1\right\}} \chi_{\{h>0\}} \\
+\int_{t_{1}}^{t_{2}} \int_{B} \chi_{\left\{u_{\epsilon}>1\right\}} \chi_{\{h>0\}},
\end{gathered}
$$

to get

$$
\int_{t_{1}}^{t_{2}} \int_{B} u \chi_{\left\{u_{\epsilon}>1\right\}} \chi_{\{h>0\}}=\int_{t_{1}}^{t_{2}} \int_{B}(u-1) \chi_{\left\{u_{\epsilon}>1\right\}} \chi_{\{h>0\}}+\int_{t_{1}}^{t_{2}} \int_{B} \chi_{\{u>1\}} \chi_{\{h>0\}},
$$

whence

$$
\int_{t_{1}}^{t_{2}} \int_{B}(u-1) \chi_{\{h>0\}}=\int_{t_{1}}^{t_{2}} \int_{B} h \chi_{\{h>0\}},
$$

and therefore $h=\lim \left(u_{\epsilon}-1\right)_{+}>0$ implies $h=u-1$.

To complete the proof use

$$
\chi_{\left\{u_{\epsilon} \leq 1\right\}}=1-\chi_{\left\{u_{\epsilon}>1\right\}} \rightarrow 1-\chi_{\{h>0\}}=\chi_{\{h \leq 1\}}, \quad \text { a.e. in } \mathbb{R}^{n} \times(0, T) .
$$

We need the following lemma, which follows from $u \in L^{\infty}\left((0, T-\delta): L_{\mathrm{loc}}^{1}\left(\mathbb{R}^{n}\right)\right)$, $\forall \delta>0$. 
3.2. Lemma. Let $0 \leq u \in L_{\text {loc }}^{1}\left(\mathbb{R}^{n} \times(0, T)\right)$. Then $u$ is a distributional solution to the Cauchy problem for (1.1) such that

$$
\lim _{t \downarrow 0} \int_{\mathbb{R}^{n}} u(x, t) \phi(x) d x=\int_{\mathbb{R}^{n}} \phi(x) d \mu(x), \quad \forall \phi \in C_{0}^{\infty}\left(\mathbb{R}^{n}\right)
$$

if and and only if $u$ is a solution to (1.1) in the sense of conservation laws, i.e.,

$$
\int_{0}^{T} \int_{\mathbb{R}^{n}}\left[u \phi_{t}+(u-1)_{+} \Delta \phi\right] d x d t+\int_{\mathbb{R}^{n}} \phi(x, 0) d \mu(x)=0,
$$

$\forall \phi \in C_{0}^{\infty}\left(\mathbb{R}^{n} \times(-\infty, T)\right)$.

To see that $u(x, t)=\lim _{\epsilon \rightarrow 0} u_{\epsilon}(x, t)$ satisfies (3.1), take $\phi \in C_{0}^{\infty}\left(\mathbb{R}^{n} \times(0, T)\right)$ and let $R>0$ such that supp $\phi(., t) \subset B_{R}, \forall t>0$. From the uniform integrability in $t$ of $\int_{B_{r}} w_{\epsilon} d x$ we get

$$
\lim _{\delta \rightarrow 0} \int_{0}^{\delta} \int_{\mathbb{R}^{n}} u_{\epsilon}\left|\phi_{t}\right| d x d t=0
$$

and

$$
\lim _{\delta \rightarrow 0} \int_{0}^{\delta} \int_{\mathbb{R}^{n}}\left(u_{\epsilon}-1\right)_{+}|\Delta \phi| d x d t=0
$$

uniformly in $\epsilon$. From the discussion above also for any $\delta>0$,

$$
\int_{\delta}^{T} \int_{\mathbb{R}^{n}} u_{\epsilon} \phi_{t} d x d t \rightarrow \int_{\delta}^{T} u \phi_{t} d x d t
$$

and

$$
\int_{\delta}^{T} \int_{\mathbb{R}^{n}}\left(u_{\epsilon}-1\right)_{+} \Delta \phi d x d t \rightarrow \int_{\delta}^{T}(u-1)_{+} \Delta \phi d x d t, \quad \epsilon \rightarrow 0 .
$$

This shows that $u$ solves (3.1). Then by the uniqueness result in $[\mathrm{K}]$ this limit must be $u_{f+\mu}$. Now $\left(u_{f+\mu_{\epsilon}}-1\right)_{+} \leq w_{\left(f+\mu_{\epsilon}-1\right)_{+}} \leq w_{(f-1)_{+}+\mu_{\epsilon}}$, letting $\epsilon \rightarrow 0,\left(u_{f}-1\right)_{+} \leq$ $\left(u_{f+\mu}-1\right)_{+} \leq w_{(f-1)_{+}+\mu}$ a.e. in $\mathbb{R}^{n} \times(0, T)$, where $w_{g}$ is the solution to the heat equation with initial datum $g$. But

$$
\lim _{(x, t) \in \Gamma_{\alpha}^{h}\left(x_{0}\right),(x, t) \rightarrow x_{0}} w_{(f-1)_{+}+\mu}(x, t)=\left(f\left(x_{0}\right)-1\right)_{+}
$$

for a.e. $x_{0} \in \mathbb{R}^{n}$, whence the result follows.

\section{Counterexample}

The following example shows that $\lim _{(x, t) \in \Gamma_{\alpha}^{h}\left(x_{0}\right),(x, t) \rightarrow x_{0}} u(x, t)$ need not exist:

4.1. Example. To construct a function $u_{I}: \mathbb{R} \rightarrow\{0,1\}$, supp $u_{I} \subset[0,1]$, such that $u_{I}$ is discontinuous at every point of a set $D \subset[0,1],|D|>0$, with osc $u_{I}(x)=$ $1, \forall x \in D$, let $\mathcal{F}=\left\{I_{\lambda}, \lambda \in \mathbb{N}\right\}$ be the family of all subintervals of $[0,1]$ having rational endpoints. Let $0<\epsilon<1$. From each $I_{\lambda}$ subtract a subinterval $\tilde{I}_{\lambda}$ with length $<\epsilon 2^{-\lambda}$. Let $E=\bigcup_{\lambda} \tilde{I}_{\lambda}$. If the $\tilde{I}_{\lambda}$ are open so is $E$, and $|E| \leq \sum_{\lambda \in \mathbb{N}} \epsilon 2^{-\lambda}=$ $\epsilon,|[0,1] \backslash E| \geq 1-\epsilon$. Define the function $u_{I}(x)=\chi_{([0,1] \backslash E)}, u_{I}$ is well defined, and $\operatorname{osc} u_{I}=1$. For $x_{0} \in[0,1] \backslash E, u_{I}\left(x_{0}\right)=1$, and since $E$ is dense in $[0,1]$, there is a sequence $x_{n} \rightarrow x_{0}$ such that $u_{I}\left(x_{n}\right)=0$. Of course, $u(x, t) \equiv u_{I}(x)$ is a 
distributional solution to (1.1). Then

$$
\lim _{(x, t) \in \Gamma_{\alpha}^{h}\left(x_{0}\right),(x, t) \rightarrow x_{0}} u(x, t)=\lim _{x \rightarrow x_{0}} u_{I}\left(x_{0}\right),
$$

which does not exist.

4.2. Remark. However it can be shown that if $\mu=f+\mu_{s}$ where $f: \mathbb{R}^{n} \rightarrow \mathbb{R}$ is lower semicontinuous, then

$$
\lim _{(x, t) \in \Gamma_{\beta}^{k}\left(x_{0}\right),(x, t) \rightarrow x_{0}} u(x, t)=f\left(x_{0}\right) .
$$

\section{REFERENCES}

[AK] D. Andreucci, M.K. Korten, Initial traces of solutions to a one-phase Stefan problem in an infinite strip, Rev. Mat. Iberoamericana, Vol. 9, No. 2 (1993), 315-332. MR 94m:35319

[B] J. E. Bouillet, Signed solutions to diffusion-heat conduction equations, Free Boundary Problems: Theory and Applications, Proc. Int. Colloq. Irsee/Ger. 1987, Vol. II, Pitman Res. Notes Math. Ser. 186 (1990), 480-485.

[BKM] J. E. Bouillet, M. K. Korten and V. Márquez, Singular limits and the "Mesa" problem, Rev. Union Mat. Argentina, Vol. 41 (1998), no. 1, 27-40.

[C] A. P. Calderón, On the behaviour of harmonic functions at the boundary, Trans. Amer. Math. Soc. 68 (1950), 47-54. MR 11:357e

[DFK] B. E. J. Dahlberg, E. Fabes and C. E. Kenig, A Fatou theorem for solutions of the porous medium equation, Proc. Amer. Math. Soc. 91 (1984), 205-212. MR 85e:35064

[DB] E. DiBenedetto, Continuity of weak solutions to certain singular parabolic equations, Ann. Mat. Pura Appl. (4), CXXX (1982), 131-176. MR 83k:35045

[H] K. M. Hui, Fatou theorem for the solutions of some nonlinear equations, Jl. Math. Anal. Applic. 183 (1994), 37-52. MR 95c:35125

[K] M. K. Korten, Non-negative solutions of $u_{t}=\Delta(u-1)_{+}$: Regularity and uniqueness for the Cauchy problem, Nonl. Anal., Th., Meth. and Appl, Vol. 27, No. 5 (1996), 589-603. MR 97h:35089

Departamento de Matemática, Facultad de Ciencias Exactas y Naturales, Universidad de Buenos Aires, Pab. No. 1, Ciudad Universitaria, 1428 Buenos Aires, Argentina

E-mail address: mkorten@dm.uba.ar

Instituto Argentino de Matemática (CONiCET), SaAvedra 15, 3ER. Piso, 1083 Buenos Aires, Argentina

Current address: Department of Mathematics, University of Liousville, Louisville, Kentucky 40292

E-mail address: korten@louisville.edu 